Brit. Heart F., 1969, 31, 246.

\title{
Effects of Surgical Closure of Ventricular Septal Defects upon Pulmonary Vascular Disease
}

\author{
K. A. HALLIDIE-SMITH, A. HOLLMAN*, W. P. CLELAND, H. H. BENTALL, \\ AND J. F. GOODWIN \\ From the Departments of Medicine (Clinical Cardiology) and Surgery (Cardio-Thoracic Unit), Royal \\ Postgraduate Medical School, and Hammersmith Hospital, London W.12
}

The indications for surgical closure of ventricular septal defects have recently been the subject of interest and discussion. It is generally agreed that the presence of pulmonary vascular disease constitutes an indication for closure, the medical prognosis of severe pulmonary vascular disease being poor (Bloomfield, 1964). There is less agreement as to the criteria for selection of patients with ventricular septal defects and pulmonary vascular disease for operation, partly because of the constantly improving techniques of operation and postoperative care, and partly because the long-term results of the closure of such defects are known in only a comparatively small number of patients followed over a relatively short period. Additional confusion has been caused by the differing interpretations of the definition of pulmonary hypertension in relation to ventricular septal defect, and by increased awareness of the difficulties in interpreting haemodynamic data, in view of the lability of the pulmonary vascular bed in children.

We are therefore presenting our experience of 42 patients with ventricular septal defect and pulmonary vascular disease, describing the patients selected and setting out our follow-up studies on 25 of these patients, who have been followed for up to 8 years after operation.

We have concentrated upon the importance of the clinical signs of pulmonary vascular disease in determining the indications for operation.

\section{Patients and Methods}

Our criteria of acceptance for operation of patients with ventricular septal defect and pulmonary vascular

Received August 27, 1968.

$\star$ Present address: Cardiac Department, University College Hospital, Gower Street, London W.C.1. disease have changed somewhat over the years, being affected both by the reduction in operative mortality and by increasing experience of the post-operative results.

Evidence for pulmonary vascular disease was based in every patient upon the clinical examination, supported by electrocardiography, radiography of the heart and lungs, and cardiac catheterization and angiocardiography. An increased pulmonary vascular resistance was diagnosed clinically if the right ventricle was obviously enlarged, pulmonary valve closure was accentuated, and the systolic murmur at the left sternal edge was ejection in type, and often accompanied by a systolic click. Clinical evidence of a left-to-right shunt (consisting of an ill-sustained left ventricular impulse, a mitral diastolic flow murmur, and electrocardiographic evidence of diastolic overload of the left ventricle) was an essential requirement for operation.

While many of these signs could have been due to a large left-to-right shunt through a large septal defect with pulmonary systolic pressure at systemic level and a low vascular resistance, the presence of pulmonary vascular disease was indicated by the character of the systolic murmur, signs of appreciable right ventricular hypertrophy on the cardiogram, and modest rather than considerable left ventricular enlargement. Confirmation of pulmonary vascular disease was obtained by cardiac catheterization, a pulmonary vascular resistance of not less than 8 units being necessary for the diagnosis of pulmonary vascular disease.

No patients were accepted who were cyanosed at rest or who had a dominant right-to-left shunt at the time of catheterization. Thus, the patients in this study all had a pulmonary artery systolic pressure at or less than 10 $\mathrm{mm}$. $\mathrm{Hg}$ below the systemic, a pulmonary arteriolar resistance of 8 units or above, and a ratio of pulmonary to systemic flow of 2.5 to 1 or less.

This group of patients comprised 42 out of a total of 227 whose ventricular septal defects were surgically repaired between 1958 and 1967 . Of these patients, 36 had "anatomically uncomplicated" defects, which have been defined as single or multiple ventricular septal 
defects, alone or in conjunction with an ostium secundum atrial septal defect or small patent ductus arteriosus. Six patients had "anatomically complicated" defects; mitral incompetence in 3, mitral stenosis in 1, aortic regurgitation in 1 , and an unsuspected large patent ductus in 1 . The patients to be described are the 36 patients with "anatomically uncomplicated" ventricular septal defects.

\section{PRE-OPERATIVE Findings}

The ages of the 36 patients at the time of operation varied between 3 and 12 years, with a mean of $7 \cdot 2$ years. Heart disease had been diagnosed between a few days of age and 2 years. The common presenting symptoms were feeding problems, failure to thrive, and frequent respiratory infections in infancy. In childhood they were active and energetic but often unable to sustain exertion as compared with other children of their own age, and some were short of breath on effort. Three children were thought to be cyanosed on effort, but none were cyanosed at rest, and only 5 showed desaturation on effort of more than 5 per cent on ear oximetry.

The children were small for their age, particularly in regard to weight (Fig. 1), 20 being below the third percentile. Some bulging of the sternum, and of the left side of the chest, was common.

On auscultation, the systolic murmur at the left sternal edge was short and soft in the majority, though in 4 children it was loud and long. The second sound was usually narrowly split with invariable accentuation of the pulmonary component. An apical third heart sound and a mid-diastolic murmur were heard in all the patients. A pulmonary systolic click was heard in 28.

Phonocardiography. This confirmed the auscultatory findings (Fig. 2) in the majority of patients. However, in only 3 patients was the systolic murmur pansystolic, but a systolic click was clearly demonstrated in all except 2 patients.

Electrocardiography. All the patients showed right ventricular hypertrophy of varying severity, and there was also some evidence of left ventricular hypertrophy in all, using the criteria of DuShane et al. (1960). The frontal plane axis varied from +90 to +150 in all except one patient whose axis was $-30^{\circ}$. Eleven patients showed evidence of right atrial hypertrophy and 3 of left atrial hypertrophy.

Radiography of the Chest. There was some generalized cardiomegaly in all the patients, mainly right-sided. The main pulmonary artery was

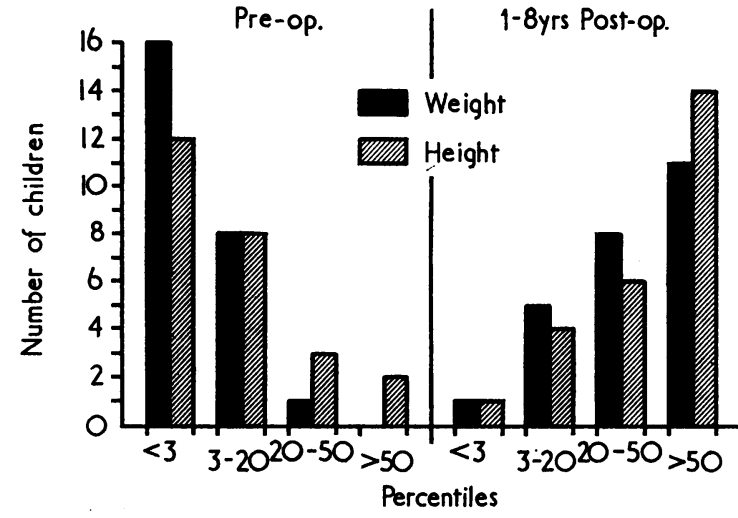

FIG. 1.-Weight and height percentile charts, showing the increase, particularly in weight, after closure of the ventricular septal defect (Standards of J. M. Tanner and R. H. Whitehouse, Institute of Child Health, London).

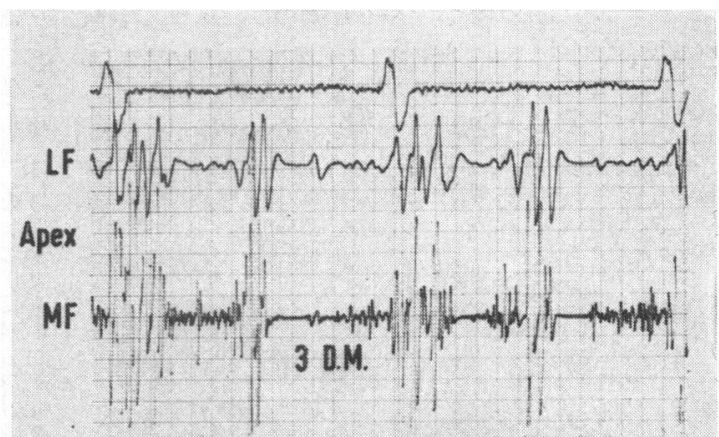

J.P.L. PHOMOCARDIOGRAM 1967

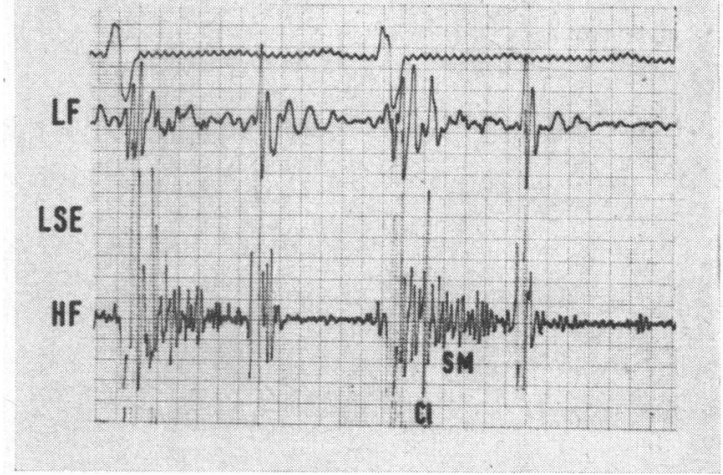

FIG. 2.-Representative phonocardiogram: pulmonary area, above, and apex, below. Time marker is $0.2 \mathrm{sec}$; $\mathrm{SM}$, systolic murmur; Cl, click; D.M., diastolic murmur; 3, third heart sound. 
TABLE

SUMMARY OF PRE-OPERATIVE, OPERATIVE, AND POST-OPERATIVE HAEMODYNAMIC DATA OF

HAEMODYNAMIC AND SURGICAL FINDINGS OF 36 PATIENTS WITH

\begin{tabular}{|c|c|c|c|c|c|c|c|c|c|c|c|c|}
\hline \multirow{2}{*}{$\begin{array}{l}\text { Case } \\
\text { No. }\end{array}$} & \multirow[b]{2}{*}{$\begin{array}{c}\text { Date } \\
\text { of } \\
\text { op. }\end{array}$} & \multirow{2}{*}{$\begin{array}{l}\text { Age (yr.) } \\
\text { and } \\
\text { sex }\end{array}$} & \multicolumn{7}{|c|}{ Pre-operative findings } & \multicolumn{3}{|c|}{ Operative findings } \\
\hline & & & Art. & $\begin{array}{l}\mathrm{P} / \mathrm{S} \\
\text { flow } \\
\text { ratio }\end{array}$ & $\underset{(1 . / \mathrm{min} .)}{\mathrm{CO}}$ & $\left|\begin{array}{c}\mathbf{R A} \\
(\mathbf{m m} . \mathbf{H g})\end{array}\right|$ & $\underset{(\mathrm{mm} . \mathbf{H g})}{\mathbf{P C}}$ & $\begin{array}{c}\text { PAR } \\
\text { (units) }\end{array}$ & $\mathbf{R p} / \mathbf{R s}$ & Anatomy & $\mid \begin{array}{c}\text { Final } \\
\text { PA } \\
\text { (mm. Hg) }\end{array}$ & $\begin{array}{c}\text { Final } \\
\mathbf{P p} / \mathbf{P s}\end{array}$ \\
\hline 1 & 1958 & $10 M$ & 93 & $2 \cdot 2$ & $4 \cdot 0$ & 2 & 13 & 8 & 0.46 & $2 \mathrm{~cm}$. VSD & $30 / 15$ & 0.23 \\
\hline 2 & 1958 & $7 \mathbf{M}$ & 92 & 1.9 & $4 \cdot 2$ & 3 & 10 & 8 & 0.5 & $\begin{array}{l}1.5 \mathrm{~cm} \text {. VSD; } \\
\text { direct }\end{array}$ & $42 / 35$ & 0.72 \\
\hline 3 & 1958 & $4 F$ & 93 & $\begin{array}{r}1.5 \\
+\quad\end{array}$ & $4 \cdot 4$ & 6 & 10 & 9 & 0.5 & $\begin{array}{l}1.25 \mathrm{~cm} \text {. VSD, } \\
2.5 \mathrm{~cm} \text {. } \\
\text { ASD direct }\end{array}$ & $40 / 30$ & 0.5 \\
\hline 4 & 1958 & $10 M$ & 91 & 1.5 & $3 \cdot 8$ & 4 & 10 & 9 & 0.55 & $\begin{array}{l}\text { suture } \\
2.5 \times 2 \mathrm{~cm} \text {. } \\
\text { VSD, direct } \\
\text { suture }\end{array}$ & $35 / 12$ & 0.41 \\
\hline 5 & 1959 & $5 M$ & 97 & 1.5 & $3 \cdot 8$ & 4 & 12 & 18 & $0 \cdot 77$ & $\begin{array}{l}\text { Multiple } \\
\text { VSD's vary- } \\
\text { ing from } 0.5 \\
\text { to } 2 \mathrm{~cm} \text {. } \\
\text { (Swiss } \\
\text { cheese) } \\
2.5 \mathrm{~cm} \text {. VSD; }\end{array}$ & $35 / 15$ & 0.31 \\
\hline 7 & 1959 & $5 F$ & 91 & 1.5 & $4 \cdot 5$ & 5 & 11 & 13 & 0.7 & $\begin{array}{l}3 \times 2 \mathrm{~cm} \text {. } \\
\text { VSD; direct }\end{array}$ & $40 / 20$ & 0.40 \\
\hline 8 & 1959 & $12 M$ & 95 & $1 \cdot 7$ & $4 \cdot 0$ & 6 & 16 & 8 & 0.45 & $\begin{array}{l}\mathrm{cm} \text {. ASD } \\
1 \mathrm{~cm} \text {. PDA } \\
\text { ligated; } \\
2 \mathrm{~cm} \text {. VSD } \\
\text { patched }\end{array}$ & $62 / 30$ & 0.88 \\
\hline 9 & 1959 & $10 \mathrm{~F}$ & 98 & $1 \cdot 3$ & $4 \cdot 2$ & 2 & 12 & 8 & 0.55 & $2.5 \mathrm{~cm}$. VSD; & $45 / 25$ & 0.35 \\
\hline 10 & 1960 & $8 M$ & 95 & $2 \cdot 0$ & $4 \cdot 0$ & 4 & 10 & 8 & $0 \cdot 40$ & $\begin{array}{l}2 \text { direct suture } \\
\text { patched }\end{array}$ & $50 / 25$ & $0 \cdot 4$ \\
\hline 11 & 1960 & $12 \mathrm{M}$ & 95 & $1 \cdot 7$ & $3 \cdot 2$ & 4 & 12 & 9 & 0.45 & $\begin{array}{l}3 \mathrm{~cm} \text {. cushion } \\
\text { type } \\
\text { patched }\end{array}$ & $30 / 15$ & 0.27 \\
\hline 12 & 1960 & $5 M$ & 96 & $1 \cdot 8$ & $4 \cdot 2$ & 1 & 10 & 12 & 0.76 & $2 \mathrm{~cm}$. cushion & $40 / 15$ & 0.26 \\
\hline 13 & 1961 & $6 \mathrm{~F}$ & 92 & 1.9 & $4 \cdot 2$ & 3 & 10 & 8 & 0.5 & $\begin{array}{l}\text { patched } \\
2 \mathrm{~cm} \text {. cushion } \\
\text { type VSD }\end{array}$ & $50 / 25$ & 0.50 \\
\hline 14 & 1961 & $10 M$ & 93 & $1 \cdot 7$ & $4 \cdot 3$ & 7 & 15 & 10 & 0.6 & $\begin{array}{l}\text { patched } \\
2.5 \mathrm{~cm} \text {. VSD } \\
\text { patched }\end{array}$ & $35 / 15$ & 0.24 \\
\hline 15 & 1962 & $5 \mathrm{~F}$ & 95 & $2 \cdot 0$ & $4 \cdot 0$ & 5 & 14 & 14 & 0.6 & $\begin{array}{l}2.5 \mathrm{~cm} \text {. direct } \\
\text { suture }\end{array}$ & $40 / 15$ & 0.50 \\
\hline 16 & 1962 & $6 F$ & 92 & $2 \cdot 5$ & $3 \cdot 3$ & 4 & 6 & 8 & 0.38 & $\begin{array}{l}1 \mathrm{~cm} . \text { VSD, } \\
2 \cdot 25 \mathrm{~cm} \text {. } \\
\text { ASD, direct }\end{array}$ & $40 / 20$ & 0.56 \\
\hline 17 & 1963 & $11 \mathrm{~F}$ & 94 & $1 \cdot 3$ & $4 \cdot 4$ & 7 & 12 & 12 & 0.64 & $\begin{array}{l}\text { suture } \\
2 \times 1 \mathrm{~cm} \text {. } \\
\text { cushion type } \\
\text { VSD }\end{array}$ & $50 / 20$ & 0.50 \\
\hline 18 & 1963 & $5 F$ & 96 & 1.6 & 3.0 & 7 & 10 & 10 & 0.55 & $\begin{array}{l}\text { patched } \\
4 \times 3 \mathrm{~cm} . \\
\text { VSD, in- } \\
\text { complete } \\
\text { common } \\
\text { ventricle, } \\
\text { patched }\end{array}$ & $50 / 35$ & 0.46 \\
\hline 19 & 1964 & $7 M$ & 90 & $1 \cdot 1$ & $2 \cdot 8$ & 6 & 12 & 17 & 0.88 & $\begin{array}{l}3 \text { VSD }(1.5 ; \\
0.5 ; 0.3 \mathrm{~cm} .) \\
\text { patched and } \\
\text { direct suture }\end{array}$ & $40 / 25$ & 0.30 \\
\hline 20 & 1965 & $6 M$ & 95 & $2 \cdot 1$ & $3 \cdot 1$ & 3 & 9 & 10 & 0.75 & $\begin{array}{l}2 \times 1.5 \mathrm{~cm} \\
\text { VSD } \\
\text { patched }\end{array}$ & $30 / 10$ & 0.43 \\
\hline 21 & 1965 & $5 F$ & 96 & $1 \cdot 7$ & $4 \cdot 0$ & 5 & 7 & 10 & 0.66 & $\begin{array}{l}2.5 \times 2.5 \mathrm{~cm} . \\
\text { VSD } \\
\text { cushion } \\
\text { type, } \\
\text { patched }\end{array}$ & $50 / 30$ & 0.47 \\
\hline
\end{tabular}


I

36 CHILDREN WITH VENTRICULAR SEPTAL DEFECT AND PULMONARY VASCULAR DISEASE

VENTRICULAR SEPTAL DEFECT AND PULMONARY VASCULAR DISEASE

\begin{tabular}{|c|c|c|c|c|c|c|c|c|c|c|c|}
\hline \multicolumn{12}{|c|}{ Post-operative findings } \\
\hline $\begin{array}{l}\text { Year } \\
\text { after } \\
\text { op. }\end{array}$ & $\underset{(\mathbf{m m} \cdot \mathbf{H g})}{\mathbf{P A}}$ & $\mid \begin{array}{c}\mathbf{R V} \\
(\mathbf{m m} . \mathbf{H g})\end{array}$ & $\mid \underset{(\mathbf{m m} . \mathbf{H g})}{\mathbf{S A}}$ & $\mid \begin{array}{c}\mathbf{R A} \\
(\mathbf{m m} . \mathbf{H g})\end{array}$ & $\underset{(\mathbf{m m} . \mathbf{H g})}{\mathbf{P C}}$ & $\begin{array}{l}\text { Art. } \\
\mathrm{O}_{2} \%\end{array}$ & $\underset{(1 . / \mathrm{min} .)}{\mathrm{CO}}$ & $\underset{\left(1 . / \mathrm{min} . / \mathrm{m} .{ }^{2}\right)}{\mathrm{CI}}$ & $\begin{array}{l}\text { Evidence } \\
\text { for residual } \\
\text { VSD }\end{array}$ & $\begin{array}{c}\text { PAR } \\
\text { (units) }\end{array}$ & $\mathbf{R p} / \mathbf{R s}$ \\
\hline 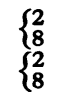 & $\begin{array}{l}40 / 11 \\
32 / 12 \\
50 / 20 \\
55 / 30\end{array}$ & $\begin{array}{l}40 / 4 \\
32 / 4 \\
50 / 6 \\
50 / 4\end{array}$ & $\begin{array}{l}110 / 80 \\
108 / 60 \\
105 / 75 \\
126 / 76\end{array}$ & $\begin{array}{l}6 \\
8 \\
1 \\
5\end{array}$ & $\begin{array}{r}11 \\
12 \\
10 \\
9\end{array}$ & $\begin{array}{l}94 \\
96 \\
94 \\
95\end{array}$ & $\begin{array}{l}5 \cdot 0 \\
7 \cdot 4 \\
3 \cdot 8 \\
5 \cdot 0\end{array}$ & $\begin{array}{l}3 \cdot 8 \\
4 \cdot 4 \\
4 \cdot 6 \\
3 \cdot 2\end{array}$ & $\begin{array}{l}\text { No } \\
\text { No } \\
\text { No } \\
\text { No }\end{array}$ & $\begin{array}{l}1 \\
1 \\
4 \cdot 5 \\
4\end{array}$ & $\begin{array}{l}0 \cdot 22 \\
0 \cdot 20 \\
0 \cdot 34 \\
0 \cdot 24\end{array}$ \\
\hline $\int 4$ & $62 / 30$ & $62 / 5$ & $120 / 70$ & 9 & 12 & 95 & 3.4 & $4 \cdot 8$ & P/S 1.3:1 & 8 & 0.5 \\
\hline$\{8$ & $50 / 12$ & $50 / 4$ & $85 / 48$ & 8 & 10 & 92 & $7 \cdot 4$ & $6 \cdot 6$ & xenon stuay & 4 & 0.3 \\
\hline$\left\{\begin{array}{l}2 \\
8\end{array}\right.$ & $\begin{array}{l}55 / 25 \\
60 / 30\end{array}$ & $\begin{array}{l}53 / 10 \\
60 / 5\end{array}$ & $\begin{array}{l}140 / 80 \\
145 / 75\end{array}$ & $\begin{array}{l}3 \\
4\end{array}$ & $\begin{array}{l}6 \\
7\end{array}$ & $\begin{array}{l}95 \\
95\end{array}$ & $\begin{array}{l}4.5 \\
6.8\end{array}$ & $\begin{array}{l}3.4 \\
4.0\end{array}$ & $\begin{array}{l}\text { No } \\
\text { No }\end{array}$ & $\begin{array}{l}6 \cdot 4 \\
6\end{array}$ & $\begin{array}{l}0.44 \\
0.50\end{array}$ \\
\hline 7 & $20 / 10$ & $20 / 4$ & $110 / 70$ & 3 & 7 & 96 & $4 \cdot 4$ & $4 \cdot 0$ & $\begin{array}{l}\text { P/S 1.2: } 1 \\
\text { indocyanine } \\
\text { green dye } \\
\text { curves; cine- } \\
\text { angiogram; } \\
\text { xenon study }\end{array}$ & $1 \cdot 6$ & $0 \cdot 18$ \\
\hline$\left\{\begin{array}{l}1 \\
7 \\
1 \\
7\end{array}\right.$ & $\begin{array}{l}32 / 8 \\
30 / 15 \\
31 / 7 \\
20 / 8\end{array}$ & $\begin{array}{l}32 / 0 \\
34 / 4 \\
32 / 0 \\
23 / 4\end{array}$ & $\begin{array}{l}110 / 75 \\
106 / 80 \\
104 / 53 \\
130 / 75\end{array}$ & $\begin{array}{l}4 \\
7 \\
3 \\
3\end{array}$ & $\begin{array}{r}10 \\
12 \\
7 \\
7\end{array}$ & $\begin{array}{l}94 \\
96 \\
94 \\
96\end{array}$ & $\begin{array}{l}5 \cdot 0 \\
7 \cdot 0 \\
3 \cdot 1 \\
5 \cdot 1\end{array}$ & $\begin{array}{l}4 \cdot 2 \\
3 \cdot 8 \\
5 \cdot 2 \\
3 \cdot 6\end{array}$ & $\begin{array}{l}\text { No } \\
\text { No } \\
\text { No } \\
\text { No }\end{array}$ & $\begin{array}{l}1 \cdot 0 \\
1 \cdot 2 \\
3 \\
1 \cdot 6\end{array}$ & $\begin{array}{l}0.28 \\
0.25 \\
0.21 \\
0 \cdot 18\end{array}$ \\
\hline$\left\{\begin{array}{l}2 \\
7\end{array}\right.$ & $\begin{array}{l}55 / 25 \\
60 / 20\end{array}$ & $\begin{array}{l}55 / 8 \\
60 / 5\end{array}$ & $\begin{array}{l}150 / 75 \\
156 / 84\end{array}$ & $\begin{array}{l}0 \\
3\end{array}$ & $\begin{array}{l}12 \\
10\end{array}$ & $\begin{array}{l}94 \\
95\end{array}$ & $\begin{array}{l}5 \cdot 5 \\
3 \cdot 3\end{array}$ & $\begin{array}{l}4 \cdot 2 \\
2 \cdot 5\end{array}$ & $\begin{array}{l}\text { P/S 1.3:1 } \\
\text { P/S 1.2:1 } \\
\text { indocyanine } \\
\text { green dye } \\
\text { curves; } \\
\text { xenon study }\end{array}$ & $\begin{array}{l}5 \\
7.6\end{array}$ & $\begin{array}{l}0.28 \\
0.35\end{array}$ \\
\hline $\begin{array}{l}\left\{\begin{array}{l}1 \\
6 \\
1 \\
6\end{array}\right.\end{array}$ & $\begin{array}{l}75 / 35 \\
80 / 20 \\
50 / 20 \\
50 / 22\end{array}$ & $\begin{array}{l}75 / 8 \\
80 / 8 \\
50 / 5 \\
45 / 5\end{array}$ & $\begin{array}{l}100 / 65 \\
100 / 60 \\
105 / 60 \\
110 / 65\end{array}$ & $\begin{array}{l}1 \\
3 \\
4 \\
6\end{array}$ & $\begin{array}{r}10 \\
8 \\
9 \\
10\end{array}$ & $\begin{array}{l}93 \\
95 \\
90 \\
94\end{array}$ & $\begin{array}{l}5 \cdot 0 \\
6 \cdot 0 \\
3.0 \\
3 \cdot 9\end{array}$ & $\begin{array}{l}4 \cdot 2 \\
3 \cdot 1 \\
4 \cdot 6 \\
3 \cdot 1\end{array}$ & $\begin{array}{l}\text { No } \\
\text { No } \\
\text { P/S } 1 \cdot 2: 1 \\
\text { indocyanine } \\
\text { green dye } \\
\text { curves, cine- }\end{array}$ & $\begin{array}{l}8 \\
6 \\
8 \\
5\end{array}$ & $\begin{array}{l}0.66 \\
0.54 \\
0.25 \\
0.28\end{array}$ \\
\hline 6 & $35 / 12$ & $36 / 7$ & $120 / 70$ & 3 & 10 & 96 & 5.9 & $4 \cdot 2$ & $\begin{array}{l}\text { angiogram } \\
\text { Indocyanine } \\
\text { green dye } \\
\text { curves; }\end{array}$ & $1 \cdot 7$ & $0 \cdot 22$ \\
\hline 6 & $40 / 18$ & $40 / 6$ & $140 / 80$ & 3 & 11 & 95 & 3.5 & $4 \cdot 2$ & $\begin{array}{l}\text { xenon study } \\
\mathrm{P} / \mathrm{S} 1 \cdot 4: 1 \\
\text { cineangiogram; }\end{array}$ & 2 & 0.22 \\
\hline $\begin{array}{l}1 \\
5\end{array}$ & $\begin{array}{l}42 / 18 \\
40 / 17\end{array}$ & $\begin{array}{l}42 / 4 \\
40 / 7\end{array}$ & $\begin{array}{l}114 / 62 \\
118 / 58\end{array}$ & $\begin{array}{l}5 \\
4\end{array}$ & $\begin{array}{r}10 \\
7\end{array}$ & $\begin{array}{l}94 \\
95\end{array}$ & $\begin{array}{l}2 \cdot 1 \\
3 \cdot 3\end{array}$ & $\begin{array}{l}3 \cdot 6 \\
3 \cdot 5\end{array}$ & $\begin{array}{l}\text { No } \\
\text { No }\end{array}$ & $\begin{array}{l}7 \\
6.5\end{array}$ & $\begin{array}{l}0.32 \\
0.36\end{array}$ \\
\hline 4 & $42 / 18$ & $42 / 6$ & $106 / 58$ & 6 & 14 & 96 & $3 \cdot 1$ & 3.9 & $\begin{array}{l}\text { P/S 1.3:1 } \\
\text { indocyanine } \\
\text { green dye } \\
\text { curves; cine- }\end{array}$ & 2 & $0 \cdot 31$ \\
\hline 4 & $32 / 12$ & $32 / 7$ & $100 / 60$ & 3 & 9 & 95 & $3 \cdot 8$ & $4 \cdot 7$ & $\begin{array}{l}\text { Indocyanine } \\
\text { green dye } \\
\text { curves, }\end{array}$ & 3 & $0 \cdot 22$ \\
\hline 4 & $35 / 10$ & $36 / 5$ & $132 / 72$ & 4 & 5 & 94 & $3 \cdot 8$ & 3.0 & $\begin{array}{l}\text { xenon study } \\
\text { Indocyanine } \\
\text { green dye } \\
\text { curves }\end{array}$ & $2 \cdot 8$ & $0 \cdot 20$ \\
\hline 3 & $55 / 25$ & $55 / 5$ & $130 / 80$ & 3 & 10 & 94 & $4 \cdot 0$ & $2 \cdot 8$ & No & $7 \cdot 5$ & 0.30 \\
\hline 3 & $65 / 29$ & $65 / 5$ & $117 / 65$ & 3 & 4 & 92 & $5 \cdot 0$ & $5 \cdot 3$ & No & $6 \cdot 6$ & 0.42 \\
\hline 1 & $50 / 20$ & $50 / 6$ & $105 / 75$ & 1 & 10 & 94 & $3 \cdot 3$ & $3 \cdot 4$ & No & 8 & 0.35 \\
\hline 2 & $25 / 10$ & $25 / 5$ & $100 / 60$ & 4 & 8 & 96 & $3 \cdot 1$ & $4 \cdot 5$ & No & $1 \cdot 2$ & 0.28 \\
\hline 1 & $44 / 12$ & $44 / 4$ & $100 / 72$ & 3 & 4 & 96 & 3.4 & $4 \cdot 2$ & $\begin{array}{l}\text { Cine angio- } \\
\text { gram, } \\
\text { xenon study }\end{array}$ & 6.4 & 0.42 \\
\hline
\end{tabular}


TABLE

\begin{tabular}{|c|c|c|c|c|c|c|c|c|c|c|c|c|}
\hline \multirow{2}{*}{$\begin{array}{l}\text { Case } \\
\text { No. }\end{array}$} & \multirow{2}{*}{$\begin{array}{c}\text { Date } \\
\text { of } \\
\text { op. }\end{array}$} & \multirow{2}{*}{$\begin{array}{l}\text { Age (yr.) } \\
\text { and } \\
\text { sex }\end{array}$} & \multicolumn{7}{|c|}{ Pre-operative findings } & \multicolumn{3}{|c|}{ Operative findings } \\
\hline & & & $\begin{array}{l}\text { Art. } \\
\mathrm{O}_{2} \%\end{array}$ & $\begin{array}{l}P / S \\
\text { flow } \\
\text { ratio }\end{array}$ & $\underset{\text { (1./min.) }}{\mathrm{CO}}$ & $\underset{(\mathbf{m m} \cdot \mathbf{H g})}{\mathbf{R A}}$ & $\underset{(\mathrm{mm} . \mathbf{H g})}{\mathbf{P C}}$ & $\begin{array}{c}\text { PAR } \\
\text { (units) }\end{array}$ & $\mathbf{R p} / \mathbf{R s}$ & Anatomy & $\begin{array}{c}\text { Final } \\
\mathbf{P A} \\
(\mathbf{m m} . \mathbf{H g})\end{array}$ & $\begin{array}{l}\text { Final } \\
\text { Pp/Ps }\end{array}$ \\
\hline 22 & 1965 & $9 \mathrm{~F}$ & 94 & $2 \cdot 0$ & $2 \cdot 8$ & 10 & 17 & 12 & 0.6 & $\begin{array}{l}2 \mathrm{~cm} \text {. cushion } \\
\text { type VSD, }\end{array}$ & $75 / 50$ & 0.60 \\
\hline 23 & 1965 & $5 M$ & 93 & $1 \cdot 2$ & $2 \cdot 8$ & 3 & 18 & 8 & 0.66 & $1.2 \mathrm{~cm}$. VSD & $78 / 40$ & 0.66 \\
\hline 24 & 1966 & $3 F$ & 91 & $1 \cdot 3$ & $4 \cdot 4$ & 10 & 14 & 9 & 0.65 & $2.5 \mathrm{~cm}$. VSD & $75 / 30$ & 0.62 \\
\hline 25 & 1967 & $7 \mathbf{M}$ & 92 & 1.5 & $3 \cdot 3$ & 8 & 14 & 14 & $0 \cdot 76$ & $\begin{array}{l}2 \times 1.5 \mathrm{~cm} . \\
\text { VSD, }\end{array}$ & $70 / 45$ & 0.66 \\
\hline 26 & 1962 & $3 F$ & 96 & $1 \cdot 3$ & $4 \cdot 0$ & 4 & 10 & 10 & 0.5 & $\begin{array}{l}2.5 \times 2.5 \mathrm{~cm} \text {. } \\
\text { VSD, direct }\end{array}$ & $40 / 20$ & $0 \cdot 25$ \\
\hline 27 & 1963 & $5 M$ & 98 & 1.5 & $3 \cdot 4$ & 3 & 12 & 10 & 0.45 & $\begin{array}{l}2 \times 2 \mathrm{~cm} \\
\text { VSD, }\end{array}$ & $50 / 10$ & 0.45 \\
\hline 28 & 1967 & $6 \mathrm{~F}$ & 95 & $1 \cdot 7$ & $3 \cdot 1$ & 3 & 9 & 11 & 0.47 & $\begin{array}{l}\text { patcnea } \\
32 \times \mathrm{cm} . \\
\text { vsD, } \\
\text { cushion } \\
\text { type, }\end{array}$ & $50 / 38$ & 0.52 \\
\hline 29 & 1958 & $8 M$ & 94 & $1 \cdot 8$ & $3 \cdot 8$ & 2 & 10 & 9 & 0.56 & 2 cm. VSD, & $110 / 50$ & $1 \cdot 0$ \\
\hline 30 & 1958 & $10 \mathrm{~F}$ & 95 & $1 \cdot 5$ & $4 \cdot 0$ & 4 & 12 & 9 & 0.50 & $2 \mathrm{~cm}$. VSD, & $90 / 50$ & $1 \cdot 0$ \\
\hline 31 & 1958 & $10 \mathrm{~F}$ & 95 & 1.5 & 3.6 & 4 & 12 & 8 & 0.55 & $2 \mathrm{~cm}$. VSD, & $100 / 50$ & $1 \cdot 0$ \\
\hline 32 & 1960 & $3 F$ & 94 & 1.5 & $4 \cdot 0$ & 2 & 10 & 8 & 0.62 & $\begin{array}{l}1.5 \times 1 \mathrm{~cm} . \\
\text { VSD, } \\
\text { patched }\end{array}$ & $90 / 60$ & $1 \cdot 0$ \\
\hline 33 & 1960 & $6 M$ & 88 & $2 \cdot 4$ & $4 \cdot 0$ & 6 & 12 & 12 & 0.45 & $\begin{array}{l}3 \text { cm. VSD, } \\
\text { cushion } \\
\text { type, } \\
\text { patched }\end{array}$ & $35 / 20$ & 0.4 \\
\hline 34 & 1962 & $8 \mathrm{~F}$ & 91 & $1 \cdot 4$ & $4 \cdot 2$ & 5 & 12 & 12 & 0.66 & $\begin{array}{l}\text { Two } 2 \mathrm{~cm} . \\
\text { VSD's, } \\
\text { direct suture }\end{array}$ & $65 / 35$ & 0.81 \\
\hline 35 & 1966 & $7 M$ & 93 & $1 \cdot 8$ & $3 \cdot 2$ & 6 & 15 & 8 & 0.52 & $\begin{array}{l}2 \times 1 \mathrm{~cm} . \\
\text { vSD, direct } \\
\text { suture; } \\
1.5 \times 1 \mathrm{~cm} . \\
\text { ASD; } 1 \mathrm{~cm} \text {. } \\
\text { PDA ligated }\end{array}$ & $70 / 55$ & 0.52 \\
\hline 36 & 1967 & $12 M$ & $\begin{array}{l}\text { (1) } 91 \\
\text { (2) } 99\end{array}$ & $\begin{array}{l}1 \cdot 4 \\
3 \cdot 0\end{array}$ & $\begin{array}{l}2 \cdot 8 \\
2 \cdot 8\end{array}$ & $\begin{array}{l}6 \\
5\end{array}$ & $\begin{array}{l}10 \\
10\end{array}$ & $\begin{array}{l}17 \\
10\end{array}$ & $\begin{array}{l}0.66 \\
0.39\end{array}$ & $\begin{array}{l}2 \times 1.2 \mathrm{~cm} . \\
\text { VSD, } \\
\text { patched }\end{array}$ & $90 / 50$ & $1 \cdot 0$ \\
\hline
\end{tabular}

Art $\mathrm{O}_{2}$, arterial oxygen saturation; P/S, ratio of pulmonary to systemic flow; CO, cardiac output; RA, right atrial mean pressure; PC, indirect left atrial mean pressure; PAR, pulmonary arteriolar resistance; Rp/Rs, ratio of pulmonary to systemic resistance; $P A$, pulmonary arterial pressure-dynamic; Pp/Ps, ratio of pulmonary to systemic arterial systolic pressure; RV, right ventricular pressure; SA, systemic arterial pressure; CI, cardiac index

prominent and there was slight or moderate pulmonary plethora, often noticeable centrally rather than peripherally (Fig. 3).

Haemodynamics. Right heart catheterization was carried out in all the patients, the majority being under heavy sedation. More recently, combined right and left heart studies have been carried out under general anaesthesia with a constant oxygen concentration of 33-40 per cent, the left heart study being carried out mainly in order to exclude a patent ductus arteriosus.

The main haemodynamic findings are shown in Tables I and II. In all the patients the pulmonary artery systolic pressure was the same as or within $10 \mathrm{~mm}$. $\mathrm{Hg}$ of the systemic arterial pressure. The ratio of pulmonary to systemic flow varied between 1.1 and 2.5 to 1 , the average being 1.3 to 1 . The pulmonary arteriolar resistance varied between 8 and 18 units with a mean of $10 \cdot 2$ units. The indirect left atrial pressure was measured in 28 of 36 patients and in 17 of these it was above $11 \mathrm{~mm}$. $\mathrm{Hg}$. The arterial oxygen saturation varied between 88 and 98 per cent (mean 93.2), 14 patients having saturations below 94 per cent. Dye dilution curves using Coomassie blue dye revealed a small right-to-left shunt shown in 5 of the patients studied in this way. The cardiac output was estimated, based on an assumed oxygen consumption, and was not corrected for body surface area, and varied between $2 \cdot 8$ and $4 \cdot 21$. $/ \mathrm{min}$. Pulmonary blood flow ranged between 3.1 and $8.81 . / \mathrm{min}$. The ratio of pulmonary to 
I-continued

\begin{tabular}{|c|c|c|c|c|c|c|c|c|c|c|c|}
\hline \multicolumn{12}{|c|}{ Post-operative findings } \\
\hline $\begin{array}{c}\text { Year } \\
\text { after } \\
\text { op. }\end{array}$ & $\underset{(\mathbf{m m} . \mathbf{H g})}{\mathbf{P A}}$ & $\begin{array}{c}\mathbf{R V} \\
(\mathbf{m m} \cdot \mathbf{H g})\end{array}$ & $\underset{(\mathbf{m m} \cdot \mathbf{H g})}{\mathbf{S A}}$ & $\underset{(\mathbf{m m} . \mathbf{H g})}{\mathbf{R A}}$ & $\underset{(\mathbf{m m} . \mathbf{H g})}{\mathbf{P C}}$ & Art. & $\underset{(1 . / \mathrm{min} .)}{\mathrm{CO}}$ & $\underset{\left(1 . / \mathrm{min} . / \mathrm{m} .{ }^{2}\right)}{\mathrm{CI}}$ & $\begin{array}{l}\text { Evidence } \\
\text { for residual } \\
\text { VSD }\end{array}$ & $\begin{array}{c}\text { PAR } \\
\text { (units) }\end{array}$ & $\mathbf{R p} / \mathbf{R s}$ \\
\hline 1 & $50 / 26$ & $50 / 8$ & $140 / 84$ & 8 & 12 & 95 & 3.4 & 3.6 & No & $7 \cdot 5$ & 0.38 \\
\hline 1 & $65 / 30$ & $65 / 6$ & $120 / 65$ & 3 & 12 & 89 & $3 \cdot 3$ & $4 \cdot 1$ & No & 10 & 0.56 \\
\hline 1 & $46 / 13$ & $46 / 4$ & $125 / 67$ & 3 & 9 & 96 & $3 \cdot 2$ & 4.8 & & 6 & 0.34 \\
\hline 1 & $60 / 21$ & $63 / 7$ & $85 / 48$ & 5 & 6 & 97 & $4 \cdot 4$ & $4 \cdot 6$ & & $7 \cdot 6$ & 0.66 \\
\hline
\end{tabular}

Well, now in U.S.A.

Well, now in Egypt

Well. less than one year since operation

Progressive low cardiac output-died

Progressive low cardiac output-died

Respiratory complications-died

Progressive low cardiac output-died

Complete heart block-died

Respiratory complications-died

Late death after second operation for aortic regurgitation

Collapsed left lung-died

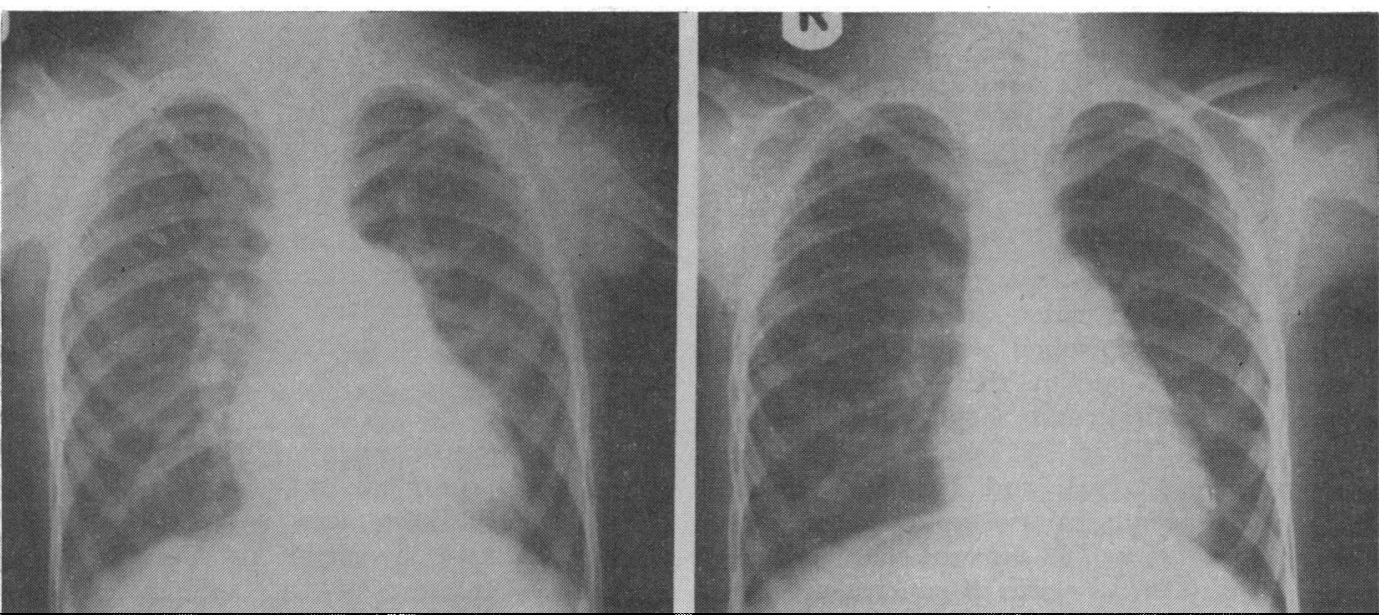

FIG. 3.-Chest $x$-rays show the reduction in over-all size of the heart and main pulmonary artery one year after successful closure of the ventricular septal defect. 
TABLE II

SUMMARY OF PRE-OPERATIVE HAEMODYNAMIC DATA IN 36 CHILDREN WITH VEN TRICULAR SEPTAL DEFECT AND PULMONARY VASCULAR DISEASE

\begin{tabular}{|c|c|c|c|c|c|}
\hline $\begin{array}{l}\text { No. of } \\
\text { patients }\end{array}$ & $\begin{array}{c}\text { Arterial } \\
\text { oxygen } \\
\text { satura- } \\
\text { tion } \\
(\%)\end{array}$ & $\begin{array}{c}\text { Ratio of } \\
\text { pul- } \\
\text { monary } \\
\text { to } \\
\text { systemic } \\
\text { flow } \\
(\mathbf{P} / \mathbf{S})\end{array}$ & $\begin{array}{c}\text { Ratio of } \\
\text { pul- } \\
\text { monary } \\
\text { to } \\
\text { systemic } \\
\text { resistance } \\
(\mathrm{Rp} / \mathrm{Rs})\end{array}$ & $\begin{array}{c}\text { Pul- } \\
\text { monary } \\
\text { arteriolar } \\
\text { resistance } \\
\text { (units) } \\
\text { PAR }\end{array}$ & $\begin{array}{c}\text { Size of } \\
\text { ventricular } \\
\text { septal } \\
\text { defect } \\
\left(\mathrm{cm} .^{2}\right)\end{array}$ \\
\hline $\begin{array}{l}28 \text { who } \\
\text { survived }\end{array}$ & $\begin{array}{c}90-98 \\
(94)\end{array}$ & $\begin{array}{c}1 \cdot 1-2 \cdot 5 \\
(1 \cdot 45)\end{array}$ & $\begin{array}{c}0.38-0.88 \\
(0.61)\end{array}$ & $\begin{array}{l}8-18 \\
(10 \cdot 6)\end{array}$ & $\underset{(3 \cdot 1)}{1 \cdot 5-12 \cdot 0}$ \\
\hline $\begin{array}{l}8 \text { who } \\
\text { did not } \\
\text { survive }\end{array}$ & $\begin{array}{c}88-98 \\
(94)\end{array}$ & $\begin{array}{c}1 \cdot 4-2 \cdot 4 \\
(1 \cdot 8)\end{array}$ & $\begin{array}{c}0.45-0.66 \\
(0.60)\end{array}$ & $\begin{array}{l}8-12 \\
(9 \cdot 4)\end{array}$ & $\underset{(1 \cdot 8)}{1 \cdot 4-2 \cdot 5}$ \\
\hline
\end{tabular}

Note: Mean values are in parentheses.

systemic resistance $(R \mathrm{p} / \mathrm{Rs})$ varied between 0.38 and 0.88 with a mean of 0.61 . Using the criteria of Kirklin (1965), 2 of our patients had mild $(\mathrm{Rp} / \mathrm{Rs} 0.25-0.45), 29$ had moderate (Rp/Rs 0.45$0.75)$, and 5 had severe (Rp/Rs 0.75 or over) pulmonary vascular disease.

In 2 patients serial cardiac catheterizations were carried out, the first being under heavy sedation and the second under general anaesthesia. The results of the first patient are shown in Table III. The second patient, a child of 6 years, was re-investigated six months after the initial test.

\section{OPERATIVE Findings}

All the ventricular septal defects were infracristal and all were large, being over $1.5 \mathrm{~cm} . / \mathrm{m} .{ }^{2} /$ B.S.A. Twenty-seven patients had a single ventricular septal defect, one being nearly a common ventricle. One patient had 2 ventricular septal defects, one 3 defects, and one had many defects (the "Swiss cheese" septum). Four patients had associated ostium secundum atrial septal defects and two patients had a small patent ductus arteriosus. Fourteen of the defects were patched and the remainder closed by direct suture.

\section{RESULTS OF OPERATION}

Mortality. Seven patients did not survive operation. An eighth patient died after a second operation, having developed aortic regurgitation after the first. In 3 patients the pulmonary artery pressure failed to fall and they developed progressive cardiac failure; 3 patients died from respiratory complications, and 1 patient, in the early days of perfusion, from complete heart block. The over-all mortality of 24 per cent compares with that of 5 per cent in our series of patients with uncomplicated ventricular septal defects without pulmonary vascular disease. However, over the past
TABLE III

RESULTS OF SERIAL CARDIAC CATHETERIZATIONS IN A PATIENT BEFORE OPERATION

\begin{tabular}{|c|c|c|c|c|c|c|}
\hline $\begin{array}{l}\text { Age } \\
\text { (yr.) }\end{array}$ & $\begin{array}{l}\text { Anaes- } \\
\text { thetic }\end{array}$ & $\begin{array}{c}\text { Arterial } \\
\text { oxygen } \\
\text { satura- } \\
\text { tion } \\
(\%)\end{array}$ & $\begin{array}{c}\text { Pul- } \\
\text { monary } \\
\text { blood } \\
\text { flow } \\
(1 . / \mathrm{min} .)\end{array}$ & $\begin{array}{c}\text { Pul- } \\
\text { monary } \\
\text { to } \\
\text { systemic } \\
\text { flow } \\
\text { ratio } \\
(\mathrm{P} / \mathrm{S})\end{array}$ & $\begin{array}{l}\text { Pul- } \\
\text { monary } \\
\text { arteri- } \\
\text { olar } \\
\text { resist- } \\
\text { ance } \\
\text { (units) } \\
\text { (PAR) }\end{array}$ & $\begin{array}{c}\text { Ratio of } \\
\text { pul- } \\
\text { monary } \\
\text { to } \\
\text { systemic } \\
\text { vascular } \\
\text { resist- } \\
\text { ance } \\
\text { (Rp/Rs) }\end{array}$ \\
\hline $\begin{array}{r}4 \\
12\end{array}$ & $\begin{array}{l}\text { Local } \\
\text { General }\end{array}$ & $\begin{array}{l}91 \\
99\end{array}$ & $\begin{array}{l}3.9 \\
8.4\end{array}$ & $\begin{array}{l}1.4 \\
3.0\end{array}$ & $\begin{array}{l}17 \\
10\end{array}$ & $\begin{array}{l}0.66 \\
0.39\end{array}$ \\
\hline
\end{tabular}

three years, with improved techniques of operation and of immediate post-operative care, the mortality in the patients with pulmonary vascular disease has fallen to 15 per cent. Of the 6 patients with "anatomically complicated" ventricular septal defects and pulmonary vascular disease previously referred to, only 1 patient survived operation.

Follow-up. The 28 children with "anatomically uncomplicated" defects and pulmonary vascular disease who survived operation have been followed up. Of them, 25 have been brought back for further study between 1 and 8 years after operation, 10 on 2 occasions. Two patients who are alive and well have not been studied for geographical reasons, while the follow-up period after operation has been too short in the final patient.

Clinical Assessment. The children were not restricted in their activities, and it was clear that both they and their parents considered them to be asymptomatic. Some indulge in such activities as competitive long distance cycling and swimming, and one is a full-time student of the French horn. Others, however, are not keen on sport, and thus it is impossible to say whether lack of inclination alone or limitation of exercise tolerance prevents them from taking full exercise.

There was a striking improvement in their general physique (Fig. 1) and this occurred particularly during the first year after operation. Bulging of the chest also resolved strikingly, particularly during the first post-operative year (Fig. 4), except in 3 patients.

Auscultatory Findings. The majority of the patients had complete right bundle-branch block after closure of their defects, and these patients had a wide fixed split of their second sound. The pulmonary component of the second sound was assessed as increased in the patients who had a residual pulmonary artery pressure of $50 \mathrm{~mm} . \mathrm{Hg}$ 


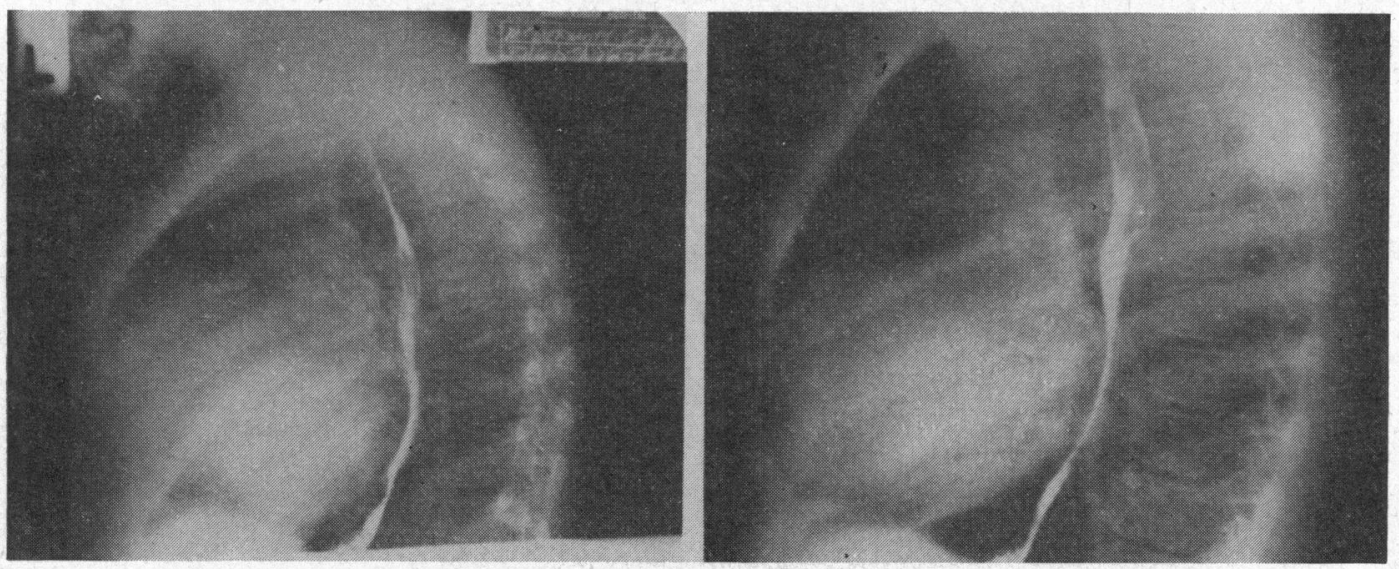

FIG. 4.-Lateral chest radiographs showing the return of chest shape to normal 8 years after successful closure of the ventricular septal defect.

or above, but was not consistently increased in those with a pulmonary artery pressure of $30-50 \mathrm{~mm}$. $\mathrm{Hg}$. An apical third heart sound was heard in 14 patients and a systolic click in 4 patients. No murmur was heard in 5 patients, soft ejection systolic murmurs occurred at the left sternal edge and pulmonary area in 16 , and loud pansystolic murmurs at the lower left sternal edge in 4 patients. The 4 patients in whom a pansystolic murmur was heard had a small residual left-to-right shunt, demonstrable on oxygen saturations and moderate residual pulmonary hypertension. The other 4 patients with residual left-toright shunts had soft ejection systolic murmurs. Only one of these had a shunt large enough to be shown on arterial oxygen saturations.

Phonocardiography. This demonstrated a systolic click in 7 patients, all of whom had some residual increases of pulmonary artery pressure; in general the clinical findings were confirmed.

Electrocardiography. Of the 25 patients, 21 developed complete right bundle-branch block and 3 patients developed left axis deviation (frontal plane axis of $-30^{\circ}$ or more). Our experience of complete right bundle-branch block with left axis deviation after the closure of ventricular septal defect, indicating interruption of the right bundle and superior branch of the left bundle, is reported elsewhere (Kulbertus, Coyne, and Hallidie-Smith, 1968).

Chest Radiography. The major changes occurred in the first year after closure of the ventricular septal defect. In all except one patient the heart became smaller and the main pulmonary arteries much less prominent (Fig. 3). Changes in the lung fields were more difficult to interpret. In some patients the pulmonary arteries remained large and gave an impression of increased pulmonary flow, despite haemodynamic proof to the contrary.

Haemodynamics. The data are set out in full in Table I, and shown graphically in Fig. 5-8.

The pulmonary artery pressure as measured on the operating table fell in all the surviving patients after closure of the ventricular septal defect. In 3 patients there was a fall of over $20 \mathrm{~mm} . \mathrm{Hg}$, and in 2 patients a rise of over $20 \mathrm{~mm}$. $\mathrm{Hg}$ at subsequent catheterization, but the table pressures were a fairly reliable guide to the subsequent pressures obtained at recatheterization. The ratio of pulmonary to systemic arterial systolic pressures as measured on the operating table was also a useful guide to subsequent measurements.

In 9 patients the pulmonary artery pressure at subsequent catheterization was within normal limits ( $30 \mathrm{~mm} . \mathrm{Hg}$ or less), in 9 patients it was between 30 and $50 \mathrm{~mm}$. $\mathrm{Hg}$, in 7 patients between 50 and 60 $\mathrm{mm}$. $\mathrm{Hg}$, and in 5 patients between 60 and 80 $\mathrm{mm}$. Hg. Of the 10 patients recatheterized a second time, the pulmonary artery systolic pressure had fallen $10 \mathrm{~mm}$. $\mathrm{Hg}$ or less in 2 patients, and had not risen by more than $10 \mathrm{~mm} . \mathrm{Hg}$ in any.

The pulmonary arteriolar resistance fell to normal limits ( 3 units or less) in 9 patients, to between 3 and 6 units in 1 patient, 6-8 units in 7 patients, and 8-10 units in 4 patients. There was no fall in 3 patients and a rise of 2 units in one patient in whom there were ventilatory difficulties during his general anaesthesia. Subsequent recatheterizations showed that the pulmonary arteriolar resistance fell by 2 


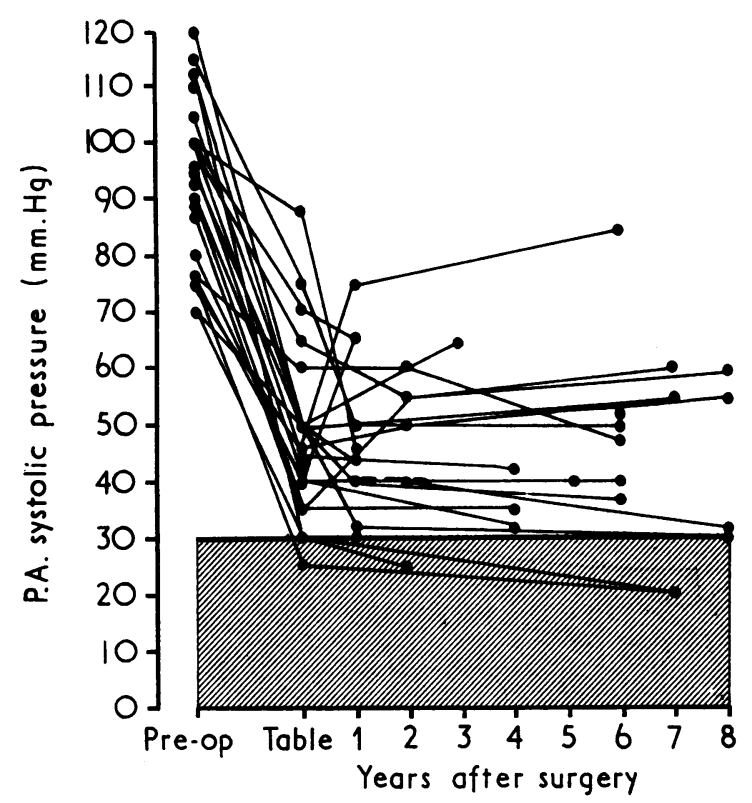

FIG. 5.-Pulmonary artery systolic pressure before and after surgical closure of ventricular septal defect in 28 children.

units or more in 2 patients and rose by 2 units or more ( 2.6 units) in one patient without any comparable rise in the pulmonary artery pressure or ratio of pulmonary to systemic resistances.

Before operation the ratio of pulmonary to systemic systolic pressures was virtually $1: 1$ in all the patients. After closure of the ventricular septal

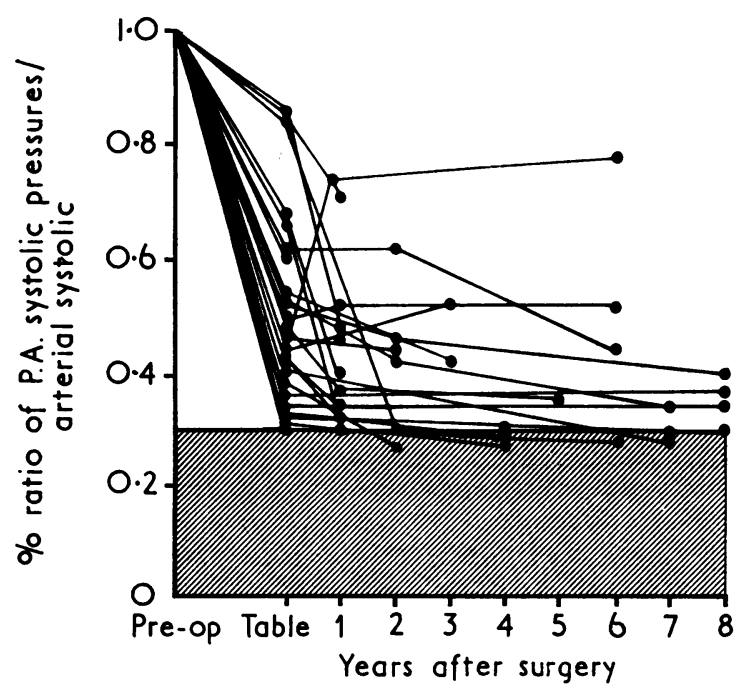

Fig. 7.-Pulmonary artery systolic pressures expressed as a percentage of systemic systolic pressure before and after surgical closure of ventricular septal defect in 28 children.

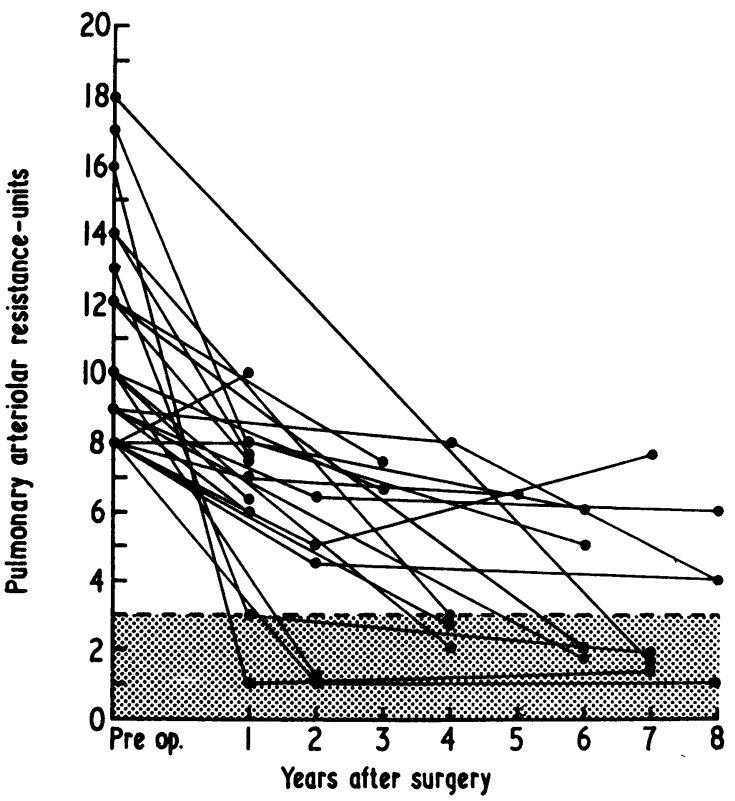

FIG. 6.-Pulmonary arteriolar resistance after closure of ventricular septal defect in 25 children.

defect the ratio of pulmonary to systemic systolic pressure fell in all the patients and reached the normal range $(0.3$ or less) in 7 . It was mildly raised $(0.3-0.5)$ in 5 , and moderately raised $(0.5-$

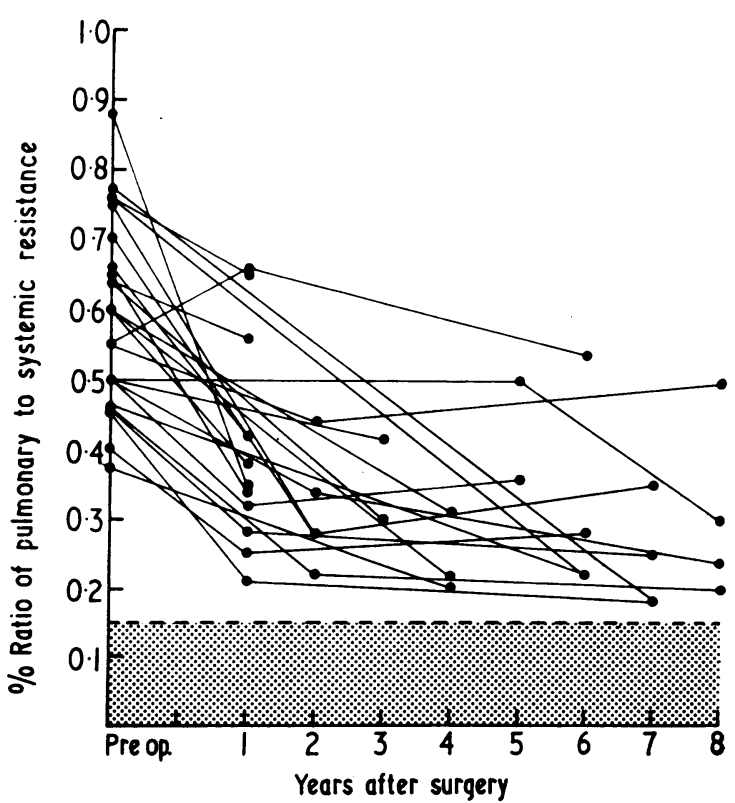

Fig. 8.-Ratio of pulmonary to systemic vascular resistance before and after surgical closure of ventricular septal defect in 25 children. 
0.75 ) in 3. There was no significant rise in any patient recatheterized, but one patient changed from the moderate to the mild group and one from the mild to normal range.

The ratio of pulmonary to systemic resistance fell in all the patients except one in whom it rose from 0.55 to 0.66 . He was recatheterized 5 years later when there was a slight fall in resistance ratio to 0.54 , and his pulmonary arterial resistance had also slightly fallen from 8 units to 6 units. Using the criteria based on the $\mathrm{Rp} / \mathrm{Rs}$ ratio, he had moderate pulmonary vascular disease before and after operation, but his measured pulmonary artery pressure of $75 / 35 \mathrm{~mm}$. $\mathrm{Hg}$ one year after operation and $80 / 20 \mathrm{~mm}$. $\mathrm{Hg} 6$ years after operation suggests that his pulmonary vascular disease is still severe. Of the 6 patients in the severe group, 4 changed to the moderate group after operation and 2 from the severe to the mild. Of the remaining 19 patients, 7 had resistance ratios below 0.25 after operation, 10 changed from the moderate to the mild range, and 2 remained in the moderate group. At subsequent catheterization there was no significant change in the ratio of pulmonary to systemic resistances.

Residual pulmonary vascular disease was also investigated using oxygen labelled carbon dioxide (West and Dollery, 1960). There was evidence of residual pulmonary hypertension in 18 of the 23 patients so examined, agreeing with the haemodynamic findings in all except 3 patients who were older children with moderate residual pulmonary vascular disease.

The indirect left atrial pressure was below $11 \mathrm{~mm}$. in all except 4 patients, the highest recorded pressure being $14 \mathrm{~mm}$.

Cardiac output, based on calculated oxygen consumption in 19 patients and measured by the Fick method in 7, was within the normal range in all patients. Arterial oxygen saturation was 94 per cent or above in all except 3 patients in whom it was 93, 92, and 89 per cent, respectively-the last patient, an asthmatic, had some difficulties with ventilation during his anaesthetic. All the patients had normal resting values on ear oximetry and did not show a fall in oxygen saturation on effort.

Residual ventricular septal defects were looked for by estimation of blood gases and by pulmonary cine-arteriography, with injection of Hypaque into the pulmonary artery with follow-through to the left side of the heart using the left anterior oblique position, in all the patients (J. M. Criley, 1963, personal communication); by central sampling with indocyanine green dye dilution curves in 12; and by measurement of pulmonary blood flow using oxygen labelled carbon dioxide in 23 . Ten patients showed evidence of a small left-to-right shunt by one or more methods of investigation (Table I), the largest ratio of pulmonary to systemic flow being 1.3:1. Of our 9 patients, 5 had a normal pulmonary vascular resistance and $\mathrm{Rp} / \mathrm{Rs}$ ratio.

Effects of Exercise. Gentle exercise was carried out on 9 patients by foot pedalling for 5 minutes. The results show (Fig. 9) that the cardiac output and arteriovenous oxygen differences rose and the pulmonary arteriolar resistance fell in all patients, and that there was no arterial oxygen desaturation. There was a rise in pulmonary artery pressure in all-being $20 \mathrm{~mm}$. or more in 4 . There was a striking rise in indirect left atrial pressure in all except one patient. In one child the left ventricular and indirect left atrial pressures were measured simultaneously and the rise in the end-diastolic pressure in the left ventricle was less than the rise in indirect left atrial pressure, giving an apparent mitral gradient of $6 \mathrm{~mm}$. More strenuous exercise was carried out in one patient who, before operation, had a pulmonary arteriolar resistance of 9 units and a pulmonary to systemic resistance ratio of 0.5 . At rest he had rapid shallow breathing with hyperventilation as indicated by a $\mathrm{PCO}_{2}$ of $35 \mathrm{~mm}$. $\mathrm{Hg}$. His pulmonary artery pressure was $55 / 37 \mathrm{~mm}$. Hg and the systemic arterial pressure $102 / 60 \mathrm{~mm}$. Hg. On exercise up to $800 \mathrm{kp} . \mathrm{m}$. there was a disproportionate increase in total ventilation and an increasing gradient between arterial oxygen saturation and $\mathrm{PO}_{2}$, suggesting a ventilation perfusion imbalance. There was an undue tachycardia with a fall in cardiac output and stroke volume. The systemic arterial pressure rose to $150 / 56 \mathrm{~mm}$. $\mathrm{Hg}$ and the pulmonary artery pressure rose to $100 / 60$ $\mathrm{mm}$. $\mathrm{Hg}$.

Drug Studies. Isoprenaline in a concentration of $0.0025 \mathrm{mg}$. $/ \mathrm{ml}$. was injected directly into the pulmonary artery of 9 patients until the heart rate rose by at least 30 beats a minute. In general they followed the same pattern as shown after mild exercise, but the individual effect was less predictable. The indirect left atrial pressure was only recorded in 3 of these patients and it rose in only one.

Acetylcholine 0.5-1 mg. was injected directly into the pulmonary artery in 7 patients, but no significant change in pressures was recorded.

\section{Discussion}

Assessment of Pulmonary Vascular Disease. The assessment of pulmonary vascular disease is essentially a histological one, as described by Heath 


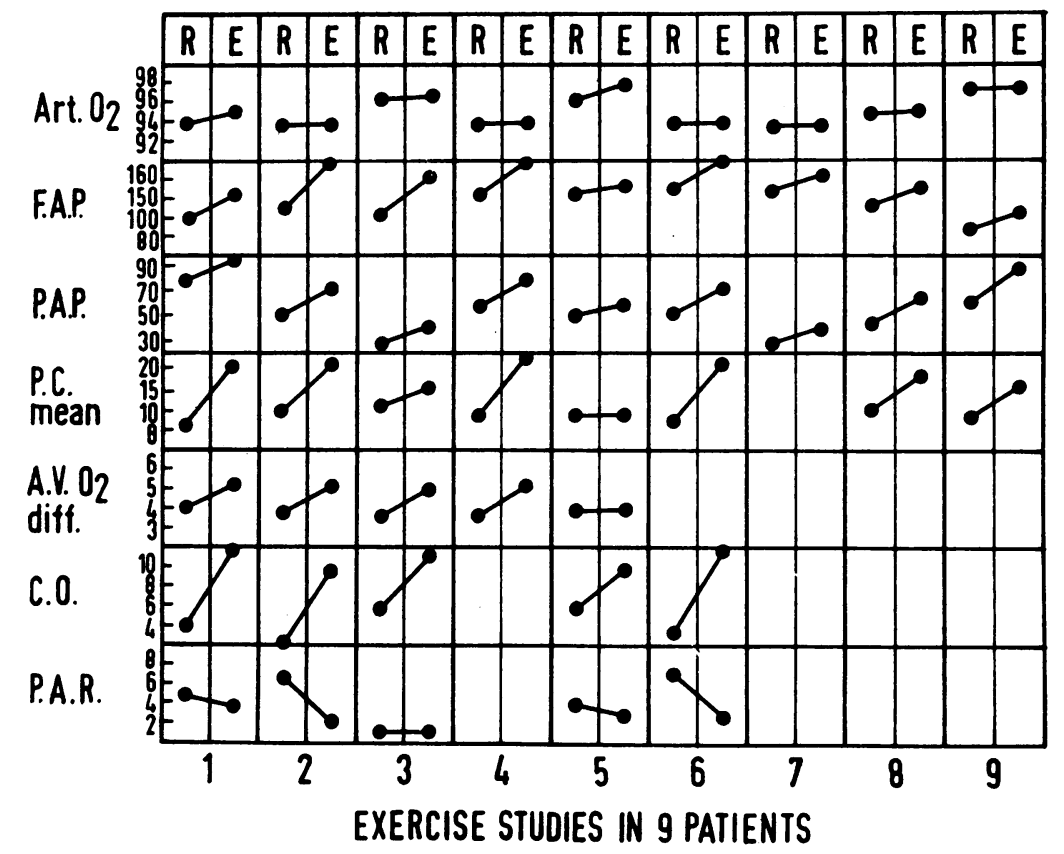

FIG. 9.-Summary of haemodynamic data obtained after mild exercise in 9 post-operative patients. The systolic pulmonary artery and systemic artery pressures are charted. (R, rest; E, exercise.) Art. $\mathrm{O}_{2}$, arterial oxygen saturation (\%); F.A.P., femoral artery pressure $(\mathrm{mm} . \mathrm{Hg})$; P.A.P., pulmonary artery pressure (mm. Hg.); P.C. mean, indirect left atrial mean pressure $(\mathrm{mm} . \mathrm{Hg})$; A.V.O $\mathrm{O}_{2}$. diff., arteriovenous oxygen difference (volumes per cent); C.O., cardiac output (1./min.); P.A.R., pulmonary arteriolar resistance (units). The numbers below refer to individual patients.

and colleagues (Heath and Edwards, 1958; Heath et al., 1958a, b), and graded according to its severity. Our findings indicate that no single clinical or haemodynamic finding can be used to assess pulmonary vascular disease. However, in the absence of lung biopsy material we can attempt to assess the pulmonary vascular disease by combining the salient clinical and haemodynamic features.

We do not use the term pulmonary hypertension in relation to the assessment of pulmonary vascular disease, since any large ventricular septal defect permits free communication between the ventricles, with transmission of systemic pressure to the pulmonary artery, whether or not there is reduced pulmonary flow consequent on increased pulmonary vascular resistance.

In the clinical assessment of pulmonary vascular disease our pre-operative findings showed that the majority of our patients had short ejection systolic murmurs, a narrowly split second heart sound, with accentuation of the pulmonary component, and a systolic ejection click. Catheterization data showed balanced or nearly balanced systemic and pul- monary artery systolic pressures, a small left-toright shunt, and a raised pulmonary vascular resistance. The length and intensity of the systolic murmur was some guide towards anticipating the severity of the pulmonary vascular disease, as was the absolute rise in the pulmonary vascular resistance. Kirklin (1965) found the most reliable guide of severity to be the ratio of pulmonary to systemic resistance. Using this criteria, 2 of our patients had mild (Rp/Rs 0.25-0.45), 29 had moderate (Rp/Rs $0.45-0.75)$, and 5 had severe $(\mathrm{Rp} / \mathrm{Rs} \mathbf{0} \cdot 75-1 \cdot 0)$ pulmonary vascular disease.

The lability of the pulmonary vascular bed in children is well known (Blount and Woodwark, 1960), and measurements of pulmonary vascular resistance may vary with the circumstances of the investigation and in particular with the oxygen tension of the inspired air (Yu et al., 1963). Thus we have placed great emphasis upon the clinical features suggesting an increased pulmonary vascular resistance in all patients as an indication of the presence of pulmonary vascular disease. These clinical features correlated well with the haemodynamic measurements under optimal conditions, 
but were at variance with measurements made under hypoxic conditions in which the pulmonary vascular resistance was spuriously high. This is illustrated in Table III, which shows that the pulmonary vascular resistance in one child was spuriously high under unsatisfactory conditions of oversedation and hypoventilation, the correct measurement being made later under general anaesthesia with full oxygenation. Similar findings were also obtained in another child, and we have been meticulous in securing optimal conditions of oxygenation at all times, usually with general anaesthesia.

Pre-operative Assessment of Patients. In assessing the patients for consideration for operation we have always rejected those who were cyanosed at rest or who did not have a dominant left-to-right shunt. We have felt that an apical mid-diastolic murmur was a valuable clinical guide to the presence of a significant left-to-right shunt, and it was heard in all our patients. We also found evidence of biventricular hypertrophy on all the electrocardiograms and some degree of cardiomegaly on radiography. A dominant left-to-right shunt was calculated on catheterization data on all our patients, but we did not regard a small right-to-left shunt as a contraindication to surgery, since some interventricular mixing is possible with any large ventricular septal defect.

Surgical Mortality. It is agreed (Lillehei et al., 1964; Hallman, Cooley, and McNamara, 1964; Kimball and McIlroy, 1966; Cartmill et al., 1966) that pulmonary vascular disease increases the hazards of surgical closure of ventricular septal defect. In published series the mortality has ranged from 15 to 45 per cent. Cartmill et al. (1966) found the mortality to be as high as 53 per cent in those patients with severe pulmonary vascular disease. Our over-all mortality was 22 per cent and depended upon many factors, such as early inexperience, alterations in technique, and improved post-operative care. We cannot with certainty relate the death rate to any one single factor (Table II) and do not find as close a correlation between pulmonary vascular resistance and mortality as did Cartmill et al. (1966), possibly because pulmonary vascular disease was less severe in most of our patients. In common with other centres, our results have improved, and over the past three years the mortality has been 15 per cent, though the numbers are small. The improved mortality is related to improved surgical techniques and postoperative care and in particular to the elimination of heart block, to ventriculotomies designed to minimize right ventricular dysfunction, to avoidance of coronary air embolism, and to attention to problems of ventilation.

In 5 of our patients the pulmonary artery pressure did not fall, and they died with a progressively diminishing cardiac output. We have histological information in only one, whose lungs showed Grade 3 changes of Heath and Edwards (1958), which are potentially reversible (Fig. 10). This patient had complete collapse of the left lung with a plug of mucus in the main bronchus. Presumably the functional capacity of the other lung was insufficient to contain his right ventricular output. The large main pulmonary artery in these patients may be a hazard by compressing the left main bronchus and producing collapse of the lung.

Heath et al. (1958a, b) have related the fall in pulmonary artery pressure and the ratio of pulmonaryto-systemic arterial systolic pressure to the histological severity of the pulmonary vascular changes. They observed that with Grade 1 changes pulmonary vascular disorder is completely reversible but with Grade 4 and 5 changes, the pulmonary artery pressure is unchanged immediately after the defect is closed. They concluded that the fall in pulmonary artery pressure immediately after closure of the ventricular septal defect represented mainly the effect of cutting off the increased flow, and possibly the relief of vasoconstriction. This being so, it is interesting to note the considerable fall in pulmonary artery pressure in some of our patients in whom the pulmonary vascular resistance before operation was considerably raised. The pulmonary vascular bed may have been capable of constricting and reducing the left-to-right shunt without undergoing severe histological changes, implying that vasoconstriction may be an important factor in the whole complex of pulmonary vascular disease. As previously noted, the pulmonary artery pressure did not change appreciably at the first and second postoperative catheterizations in these patients.

The pulmonary arteriolar resistance also fell in all except 4 patients and remained remarkably unchanged at subsequent recatheterizations. It remained raised to 5 units or above only in those with at least moderate residual pulmonary hypertension.

The ratio of pulmonary to systemic resistance showed a comparable situation. There was a striking fall in 5 of the 6 patients with initial Rp/Rs ratios of 0.75 and above, again suggestive but not conclusive evidence of a falsely high value at the initial catheterization or the absence of severe pathological changes. There was very little change in the resistance ratios in patients recatheterized a second time.

Our findings indicate a fall in pulmonary arterypressure and in the ratio of pulmonary to systemic: 


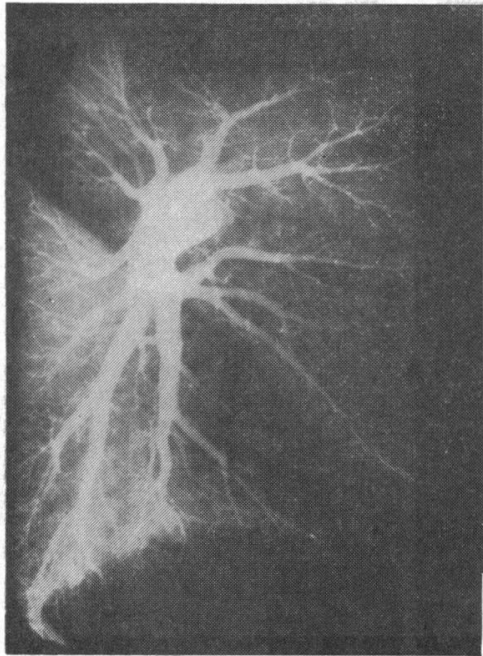

(A)

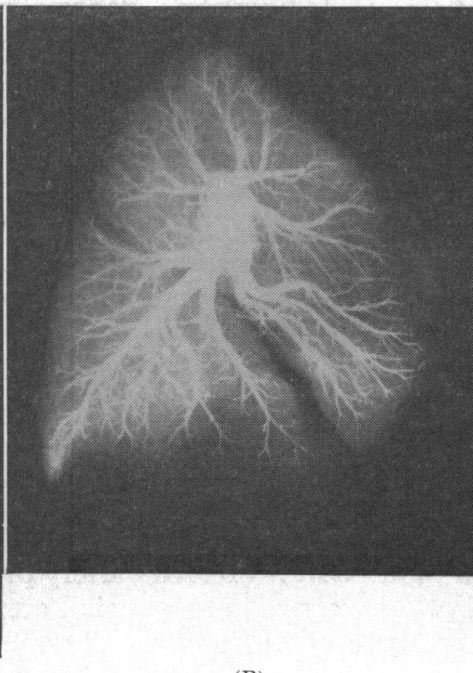

(B)

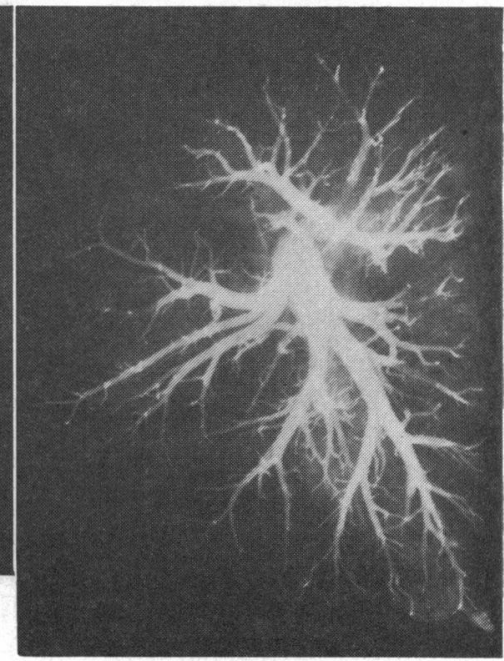

(C)

FIG. 10.-Radio-opaque contrast medium injected into post-mortem lung to show (A) normal pulmonary vasculature; (B) Grade 3 (Heath) changes in patient with a ventricular septal defect who died after postoperative collapse of the other lung; (C) advanced non-reversible histological changes in a young woman with a ventricular septal defect who was cyanosed at rest and who was not operated upon.

arterial systolic pressures on the operating table immediately after closure of the ventricular septal defect in all our patients. This initial fall has been confirmed at cardiac catheterization 1 to 2 years after operation and up to a maximum of 8 years after closure of the defect. The ratio of pulmonary to systemic vascular resistance likewise was less than before operation 1 to 2 years after operation, and the pulmonary vascular resistance was also less in all except 4 patients, with no evidence of deterioration in those subsequently recatheterized. The persistence of a small residual ventricular septal defect, contrary to previous experience (Kimball and McIlroy, 1966), did not appear to be an adverse factor. We have therefore not demonstrated any progression of pulmonary vascular disease after closure of the defect, but rather have shown a fall in pulmonary vascular resistance. We lack, however, information as to how the post-operative haemodynamic changes may be related to histological changes in the pulmonary vessels.

The majority of our patients are left with mild or moderate pulmonary vascular disease, and one patient has moderately severe residual pulmonary vascular disease by all criteria. We have no evidence that he or any of the other patients are deteriorating, but the natural history of patients with isolated pulmonary hypertension of this aetiology is unknown.
Effects of Exercise. On only very moderate exercise the response to exercise was normal except for the rise in pulmonary artery pressure (Turino, Brandfonbrener, and Fishman, 1959). This response was also obtained when an intravenous infusion of isoprenaline, which is known partially to mimic the effects of exercise, was given, but the results were less conclusive. An interesting finding was the increase in the indirect left atrial pressure on exercise which occurred in 6 of the 9 patients in whom it was measured, the resting pressures being normal. In normal subjects mild exercise would not appreciably change the pressure, and a maximum rise of 3-4 mm. could be expected on more strenuous exercise (Dexter et al., 1951). The cause of the raised indirect left atrial pressure is not clear but the pressure tracings were technically satisfactory. Possibly it did not reflect the direct left atrial pressure, or could be indicative of left ventricular failure. In one patient we obtained a left ventricular enddiastolic pressure simultaneously with the indirect left atrial pressure, and were able to show that there was an apparent mitral gradient of 5-6 mm., the left ventricular end-diastolic pressure still being within normal limits. This suggests that the indirect left atrial pressure as measured may not always be a true reflection of the direct left atrial pressure. We have no good explanation to offer, but this discrepancy has been reported previously under certain 
experimental circumstances, including anoxia, in dogs (Rivera-Estrada et al., 1958).

It is known that a high left atrial pressure may cause an increase in pulmonary arteriolar resistance in mitral stenosis (Holling, 1952), and in ventricular septal defect (Bloomfield, 1964; Hoffman and Rudolph, 1965). Left atrial hypertension is followed by a reduction in blood flow to the lower zones of the lungs (Doyle et al., 1957; Dollery et al., 1961). In the majority of our patients the indirect left atrial pressure was slightly raised before operation but was normal under basal conditions of the operation.

The increase in indirect left atrial pressure after operation on only mild effort suggests that under the circumstances of normal life the left atrial pressure might be appreciably raised for most of the day in active and symptomless children. This increase could well perpetuate pulmonary arteriolar vascularization which could militate against the regression of organic vascular obstruction. However, persistent increases in left atrial pressure should be associated with the characteristic inversion of the normal pattern of blood flow in the lungs, with reduced flow to the lower zones and increased flow to the upper zones, and we have so far no evidence that this has occurred.

The raised left atrial pressure on effort may be an indication of impaired left ventricular function, and further left ventricular function studies are required.

One patient who was more strenuously exercised responded by hyperventilation, arterial oxygen desaturation, a fall in cardiac output and stroke volume, and a considerable rise in pulmonary artery pressure.

Further studies are necessary in order to assess the response of these patients to exercise. These findings, however, and the three sudden late deaths in the series of Cartmill et al. (1966), sound a note of caution in the management of such patients, suggesting that perhaps they should be barred from competitive sports and should avoid high altitudes.

\section{SUMMARY}

The results of surgical closure of ventricular septal defect in $\mathbf{4 2}$ children with a raised pulmonary vascular resistance are presented. Six patients had anatomical complications which added to the problems of the operation: these were mitral regurgitation in 3, mitral stenosis in 1, aortic regurgitation in 1 , and a large patent ductus arteriosus in 1 . The remaining 36 patients had single or multiple ventricular septal defects alone or with a secundum atrial septal defect or small patent ductus, and were regarded as anatomically uncomplicated in that the associated lesions, when present, did not provide added surgical problems.

Evidence for a raised pulmonary vascular resistance was based upon the clinical features and upon measurements of the ratios of pulmonary to systemic resistance, and pulmonary blood flow obtained at cardiac catheterization.

Evidence for the lability of the pulmonary vascular bed and the increase in resistance under hypoxic conditions is presented, and the importance of the physical signs as a guide to pulmonary vascular disease is stressed, as is haemodynamic assessment under conditions of full oxygenation.

All the patients in the series had a left-to-right shunt (which could be detected clinically), pulmonary artery pressure at systemic level, and a pulmonary vascular resistance of not less than 8 units. The arterial oxygen saturation varied between 88 and 98 per cent. Electrocardiography showed some evidence of a left ventricular volume load in all patients. No patient who was cyanosed at rest and who did not have some clinical, auscultatory, and electrocardiographic evidence of a leftto-right shunt was accepted for operation.

Five of the six patients with anatomical complications did not survive operation. The over-all mortality in the 36 considered to be anatomically uncomplicated was 22 per cent, reduced to 15 per cent in the last 3 years.

Twenty-five patients were reinvestigated one to eight years after operation, 10 on two occasions. The results showed a fall in pulmonary artery pressure and in pulmonary vascular resistance. The majority had some residual pulmonary vascular disease, but there was no evidence of any progression over the period of the study. Mild exercise produced an increase in cardiac output, but pulmonary artery pressure and indirect left atrial pressure also rose and the reasons for these changes are discussed. The possible undesirable effects of an increase in pulmonary artery pressure on effort upon the pulmonary vascular bed are debated.

The long-term effects of continued pulmonary hypertension are still unknown, but it is felt that a surgical mortality in the region of 15 per cent is warranted in the light of the uncertain medical prognosis. In view of the encouraging long-term results so far, we believe that surgical closure is to be advised in patients with a raised pulmonary vascular resistance and clinical evidence of a dominant left-to-right shunt provided no serious anatomical complication is present.

Dr. J. M. B. Hughes (Respiratory Research Group) carried out the investigations of regional lung perfusion and we are most grateful to him for his help and interest. 
We should also like to thank Dr. R. H. T. Edwards (Department of Respiratory Physiology) for carrying out the combined respiratory and exercise study, and Drs. J. Abbott and H. Kulbertus for their assistance in the haemodynamic investigations. We are most grateful to Professor R. E. Steiner and the Department of Radiodiagnosis and to Dr. C. M. Oakley for advice and practical help. Dr. E. Olsen of the Department of Morbid Anatomy kindly provided the post-mortem angiograms for Fig. 10.

\section{REFERENCES}

Bloomfield, D. K. (1964). The natural history of ventricular septal defect in patients surviving infancy. Circulation, $29,914$.

Blount, S. G., Jr., and Woodwark, G. M. (1960). Considerations involved in the selection for surgery of patients with ventricular septal defects. Amer. $\mathcal{F}$. Cardiol., 5, 223.

Cartmill, T. B., DuShane, J. W., McGoon, D. C., and Kirklin, J. W. (1966). Results of repair of ventricular septal defect. F. thorac. cardiovasc. Surg., 52, 486.

Dexter, L., Whittenberger, J. L., Haynes, F. W., Goodale, W. T., Gorlin, R., and Sawyer, C. G. (1951). Effect of exercise on circulatory dynamics of normal individuals. f. appl. Physiol., 3, 439.

Dollery, C. T., West, J. B., Goodwin, J. F., Hugh-Jones, P., and Wilcken, D. E. L. (1961). Regional pulmonary blood flow in mitral and congenital heart disease. In CIBA Symposium on Problems of Pulmonary Circulation, p. 17. Ed. by A. V. S. de Reuck and M. O'Connor. Churchill, London.

Doyle, A. E., Goodwin, J. F., Harrison, C. V., and Steiner, R. E. (1957). Pulmonary vascular patterns in pulmonary hypertension. Brit. Heart f., 19, 353.

DuShane, J. W., Weidman, W. H., Brandenburg, R. O., and Kirklin, J. W. (1960). The electrocardiogram in children with ventricular septal defect and severe pulmonary hypertension. Circulation, 22, 49.

Hallman, G. L., Cooley, D. A., and McNamara, D. G. (1964). Surgical treatment of ventricular septal defect associated with pulmonary hypertension. $\mathcal{F}$. thorac. Cardiovasc. Surg., 48, 588.

Heath, D., and Edwards, J. E. (1958). The pathology of hypertensive pulmonary vascular disease. A description of six grades of structural changes in the pulmonary arteries with special reference to congenital cardiac septal defects. Circulation, 18, 533.

-, Helmholz, H. F., Jr., Burchell, H. B., DuShane, J. W., and Edwards, J. E. (1958a). Graded pulmonary vascular changes and hemodynamic findings in cases of atrial and ventricular septal defect and patent ductus arteriosus. Circulation, 18, 1155.

$-,-\longrightarrow,-$, , Kirklin, J. W., and Edwards, J. E. (1958b). Relation between structural changes in the small pulmonary arteries and the immediate reversibility of pulmonary hypertension following closure of ventricular and atrial septal defects. Circulation, 18, 1167.

Hoffman, J. I. E., and Rudolph, A. M. (1965). The natural history of ventricular septal defects in infancy. Amer. f. Cardiol., 16, 634.

Holling, H. E. (1952). Cardiovascular research and the surgery of the heart. Brit. med. Bull., 8, 358.

Kimball, K. G., and Mcllroy, M. B. (1966). Pulmonary hypertension in patients with congenital heart disease. Pre- and postoperative hemodynamics, pulmonary function, and criteria for surgical closure of defects. Amer. f. Med., 41, 883.

Kirklin, J. (1965). Ventricular septal defect with pulmonary vascular disease. N.Z. med. F., p. 64 (Cardiac Suppl.), 34.

Kulbertus, H., Coyne, J., and Hallidie-Smith, K. A. (1968). Left axis deviation with right bundle-branch block in ventricular septal defect before and after surgical closure. In Proceedings of the The British Cardiac Society. Brit. Heart $\mathcal{F}$., 30, 422.

Lillehei, C. W., Levy, M. J., Adams, P., and Anderson, R. C. (1964). High-pressure ventricular septal defects. $\mathcal{F}$. Amer. med. Ass., 188, 949.

Rivera-Estrada, C., Saltzman, P. W., Singer, D., and Katz, L. N. (1958). Action of hypoxia on the pulmonary vasculature. Circulat. Res., 6, 10.

Turino, G. M., Brandfonbrener, M., and Fishman, A. P. (1959). The effects of changes in ventilation and pulmonary blood flow on the diffusing capacity of the lung. 7. clin. Invest., 38, 1186.

West, J. B., and Dollery, C. T. (1960). Distribution of blood flow and ventilation-perfusion ratio in the lung, measured with radio-active carbon dioxide. $\mathcal{F}$. appl. Physiol., 15, 405.

Yu, P. N., Glick, G., Schreiner, B. F., and Murphy, G. W. (1963). Effects of acute hypoxia on the pulmonary vascular bed of patients with acquired heart disease. Circulation, 27, 541. 